\title{
A Computational Study of the Protoisomerization of Indigo and Its Imine Derivatives
}

\author{
Laurence Hajjar ${ }^{1}$, Robin G. Hicks², and Tao Zengl,* \\ ${ }^{1}$ Department of Chemistry, Carleton University, Ottawa, ON K1S5B6 \\ Canada \\ ${ }^{2}$ Department of Chemistry, University of Victoria, Victoria, BC V8W2Y2 \\ Canada
}

\begin{abstract}
The protoisomerization (isomerization induced by protonation) mechanisms of indigo as well as indigo di- and monoimine derivatives have been investigated using computational chemistry. Both density functional theory (M06-2X) and wave function theory (GMC-QDPT) methods were used to obtain reliable results. A solvation model (CPCM with $\mathrm{CH}_{2} \mathrm{Cl}_{2}$ solvent) was employed to mimic the actual environment of the isomerization. The calculations reveal that the protoisomerizations of both the indigo and its imine derivatives are thermodynamically favorable. However, the energy barriers for rotating the double bonds in the derivatives are found to be lower than the one for indigo. More importantly, the imine derivatives undergo one-step isomerization, whereas a twostep process is predicted indigo itself. The computational results provide insightful explanation for the different protoisomerization propensities of the parent indigo and its imine derivatives observed in experiment.
\end{abstract}




\section{INTRODUCTION}

The role of trans-cis isomerization around a double bond can never be overstated in organic chemistry and biochemistry and is a long lasting research subject. ${ }^{1-2}$ In an alkene system with a stable double bond, the isomerization only occurs under photoexcitation or metal catalysis. If the double bond is embedded in a conjugated system, usually, the isomerization can occur more readily. ${ }^{3,4}$ The indigo dye molecule (1, Scheme 1$)$, however, is an exception. As one of the earliest known vat dyes, indigo and its chemistry have been attracting research interest for millennia. ${ }^{5-6}$ Despite possessing a conjugated system, indigo's trans configuration about the central $\mathrm{C}=\mathrm{C}$ bond does not undergo the isomerization even under photoexcitation..$^{5,7,8} \mathrm{An}$ attempt to isomerize indigo through forming its keto-enol tautomer first also turned out unsuccessful..$^{9}$ Only when reduced to its leuco form can indigo undergo photoisomerization. ${ }^{10}$ The stability of the trans configuration of indigo structure is partially attributed to the presence of intramolecular $\mathrm{N}-\mathrm{H}$...O hydrogen bonding, ${ }^{11}$ and the efficient deactivation channel for the photoexcited state through intramolecular single proton transfer. ${ }^{12,13}$

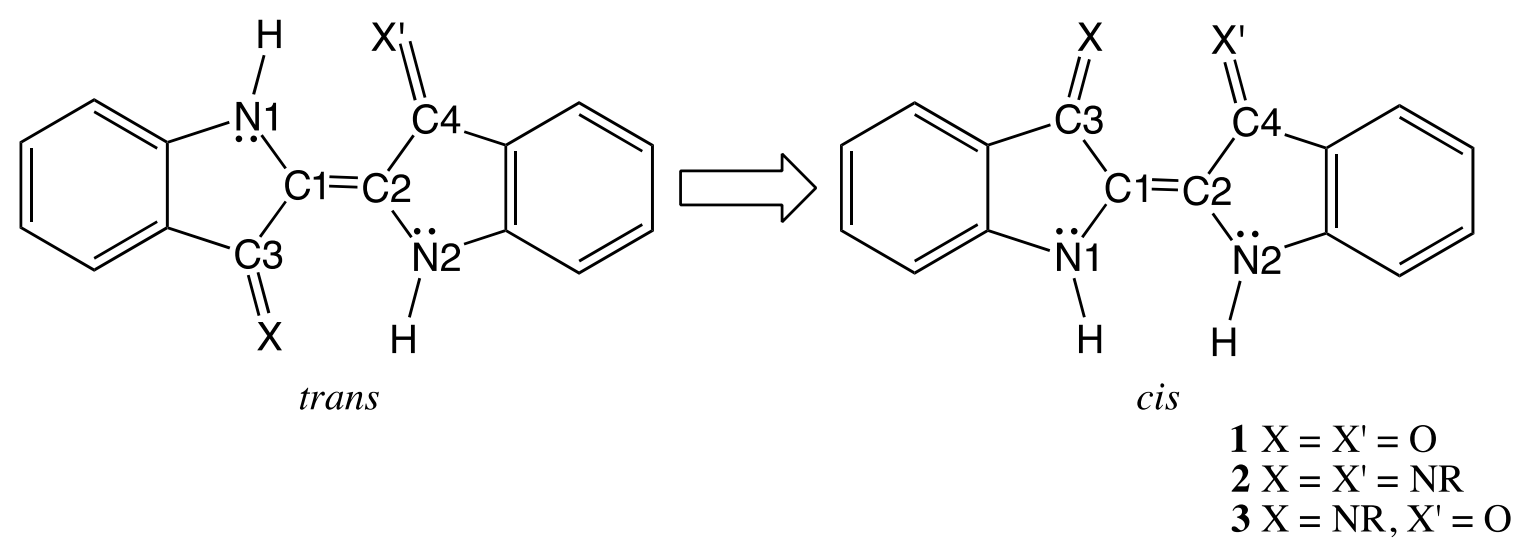

Scheme 1 General trans-to-cis isomerization of indigo and its imine derivatives.

Surprisingly, such a rigid $\mathrm{C}=\mathrm{C}$ bond framework was recently shown to be softened in the di- and monoimine derivatives of indigo ( $\mathbf{2}$ and $\mathbf{3}$ in Scheme 1, 
respectively); when the molecules are protonated in acidic environments, only their cis configurations are observed. ${ }^{14}$ Treatment of the protonated isomerized structure with a base regenerates the original trans structure, indicating a fully reversible process. This process for the indigo mono- and diimines differs from those for thioindigo ${ }^{15,16}$ and N,N'disubstituted indigo derivatives ${ }^{22-17}$ in that light is not required in the former. The similar protoisomerization was however not observed for indigo. ${ }^{20}$ Indigo and its imine derivatives' different responses to protonation motivate us to carry out the present computational study, with the objective to understand the underlying mechanism in the protoisomerization.

Aside from the quest for knowledge, there is also a practical motivation to investigate the protoisomerization. Guided by the better understanding of the isomerization, people can synthesize alternate, more efficient indigoid dyes with various properties, e.g., solubilities, colors, durabilities, capabilities as chemical indicators, etc. Also, the two N(indole)CCCN(imine) moieties in the indigo diimine (2, called "Nindigo" by Hicks and co-workers ${ }^{18}$ ) are similar to $\beta$-diketiminates (NacNacs), and hence it is a promising binucleating ligand. ${ }^{19-20}$ However, once it adopts a cis configuration, the two identical binding sites convert to one with two imine $\mathrm{N}$ atoms and one with two indole $\mathrm{N}$ atoms, which exhibit different coordination properties. ${ }^{26,21}$ Therefore, the protoisomerization provides a measure to adjust the ligation capability of Nindigo through tuning the acidity of the environment. This versatility in coordination chemistry reflects Nindigo's potential of being a switchable $\pi$ system. ${ }^{22}$ Furthermore, understanding the mechanism will improve our knowledge in controlling the configurations of indigo and its derivatives, and ultimately facilitate their applications in organic electronics. ${ }^{23}$

\section{METHODOLOGY}

Our focus is on the thermodynamics and kinetics of the trans-to-cis isomerization of the protonated indigo and its imine derivatives. Calculating the energy difference between the trans and cis configurations and the energy barrier of the double bond rotation is of 
essence. All geometry optimizations were performed using Density Functional Theory (DFT) method with the M06-2X functional. ${ }^{24}$ Closed-shell electronic configurations are reasonably assumed for all trans and cis structures. The transition state (TS) in the isomerization involves breaking the $\pi$ bond and contains a substantial diradical character in its singlet electronic state, requiring a multireference wave function description. To avoid carrying out the expensive multireference calculation in searching for the saddle point, we instead optimize the structure for the lowest triplet state to obtain the approximate TS configuration. This approximation is rooted in the characteristic potential energy curves of olefins along the torsional motion around the central $\mathrm{C}=\mathrm{C}$ bond: the minimum of the lowest triplet state corresponds to the TS structure of the singlet ground state. ${ }^{25}$ The more diradical character in the singlet TS, the more accurate the so-obtained TS structure. The triplet is described using an unrestricted open-shell scheme. There is only little spin-contamination in the unrestricted calculations: the $<\mathrm{S}^{2}>$ values $(\sim 2.03)$ only slightly deviate from the exact value of 2 . All discussed structures have their coordinates presented in Section S1 of the Supporting Information (SI).

At the optimized trans, cis, and TS structures of a species, the General MultiConfiguration Quasi-Degenerate Perturbation Theory ${ }^{26,27}$ (GMC-QDPT) method is used to calculate their energies, whose differences give the isomerization energy and the barrier. The $\pi$-interaction between the two (substituted)3-oxindole units changes most significantly in the isomerization. This interaction is largely described by the bonding character of the highest occupied and the antibonding character of the lowest unoccupied molecular orbitals (HOMO and LUMO; shown in Figure S6 in SI for protonated indigo as examples); the active space with the two orbitals and the two electrons within $(2 \mathrm{o} 2 \mathrm{e})$ is adequate to capture the electronic structure modification involved. In actual calculations, HOMO-2 and HOMO-1 are also included in the active space (i.e., a 4o6e space) to facilitate convergence.

In the experiment of Hicks and co-workers, ${ }^{20}$ the isomerization of the imine derivatives occurred in $\mathrm{CH}_{2} \mathrm{Cl}_{2}$ solutions of strong acids. We simulate the solvent environment using the conductor-like polarizable continuum model (C-PCM). ${ }^{28,29} \mathrm{We}$ 
calculate the solvation energy of a structure at the DFT level and then add this correction to the GMC-QDPT energy, i.e.:

$$
\mathrm{E}(\mathrm{GMC}-\mathrm{QDPT}+\mathrm{PCM})=\mathrm{E}(\mathrm{GMC}-\mathrm{QDPT})+\mathrm{E}(\mathrm{DFT}+\mathrm{PCM})-\mathrm{E}(\mathrm{DFT} \text {, only }) \text {. }
$$

The so-obtained energy contains proper description of dynamical and non-dynamical electron correlation, as well as solvent effects. All isomerization energies and barriers reported below are obtained using the E(GMC-QDPT + PCM), unless further specified. Since the protoisomerization propensities of the three investigated molecules are similar in different acids, the counteranions are not likely to play an active role and will not be considered in this study.

At each optimized structure, hessian calculations were carried out, and the resultant zero-point vibrational energy is included in calculating energy difference. By doing this, we have ignored the error induced by including the zero-point energy of the torsional mode around the central CC bond at the TS structure, which should have an imaginary frequency in a real TS structure optimization. The error is small as the wave number of the torsional mode is only of tens of $\mathrm{cm}^{-1}$. All calculations were carried out using the GAMESS-US program package ${ }^{30,31}$ and the cc-pVDZ basis set. ${ }^{32}$

\section{RESULTS AND DISCUSSION}

We first calibrate our methodology through comparing our optimized structures with the experimentally determined ones, using Nindigo as the model. Selected calculated bond lengths of trans Nindigo (2-trans) and its protonated cis structure (2p-cis, "p" for protonated) are summarized in Table 1, in comparison with the experimental values. To save computational resource, we have replaced the bulky groups attached to the imine nitrogen (e.g., 2,6-dimethylphenyl (dmp)) by methyl groups throughout this work. 
Table 1 Selected calculated bond lengths $(\AA)$ for $\mathbf{2}$-trans and $\mathbf{2 p}$-cis.
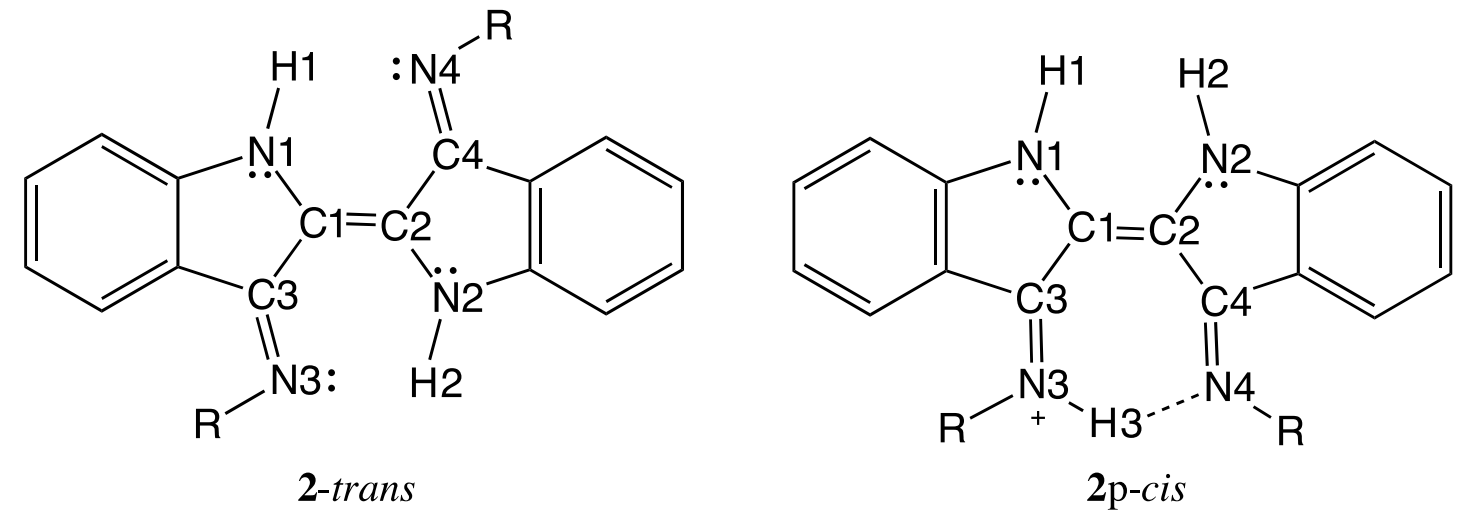

\begin{tabular}{ccccc}
\hline Bond & 2-trans & (Calc. $)$ & 2-trans $^{\mathbf{b}}\left(\right.$ Exp. $\left.^{27}\right)$ & 2p-cis \\
C1-C2 & 1.36 & $1.356(3)$ & 1.37 & $1.3800(15)$ \\
C1-C3 & 1.48 & $1.4638(17)$ & 1.46 & $1.4649(15)$ \\
C2-C4 & - & - & 1.50 & $1.4836(15)$ \\
C1-N1 & 1.38 & $1.3753(17)$ & 1.40 & $1.3881(14)$ \\
C2-N2 & - & - & 1.37 & $1.3716(15)$ \\
C3-N3 & 1.29 & $1.2939(17)$ & 1.31 & $1.3142(14)$ \\
C4-N4 & - & - & 1.28 & $1.2974(15)$ \\
\hline
\end{tabular}

${ }^{\mathrm{a}} \mathrm{R}=\mathrm{CH}_{3} .{ }^{\mathrm{b}} \mathrm{R}=2,6$-diisopropylphenyl. ${ }^{\mathrm{c}} \mathrm{R}=2,6$-dimethylphenyl.

Our calculated bond lengths deviate from the experimental values by less than $0.02 \AA$. The agreement raises our confidence on the performance of the DFT method in geometry optimization and the treatment of replacing bulky substituents on the imine $\mathrm{N}$ atoms by methyl. The isomerization energy for the 2 p-trans to $2 \mathrm{p}$-cis conversion is calculated to be -0.20 and $-0.14 \mathrm{eV}$ at the DFT level ("-": cis being more stable) for $\mathrm{R}=$ $\mathrm{CH}_{3}$ and phenyl, respectively. This key energy quantity is well preserved in the $\mathrm{R}=$ methyl simplification. A more detailed structural comparison of the $\mathrm{R}=$ methyl and phenyl for $2 p$ and the unprotonated 2 is given in Section S2 of SI. The $2 p$ structures 
calculated with and without $\mathrm{CH}_{2} \mathrm{Cl}_{2} / \mathrm{PCM}$ differ by less than $0.02 \AA$, suggesting minor solvent effects in molecular geometry. 


\section{Protonated Indigo}

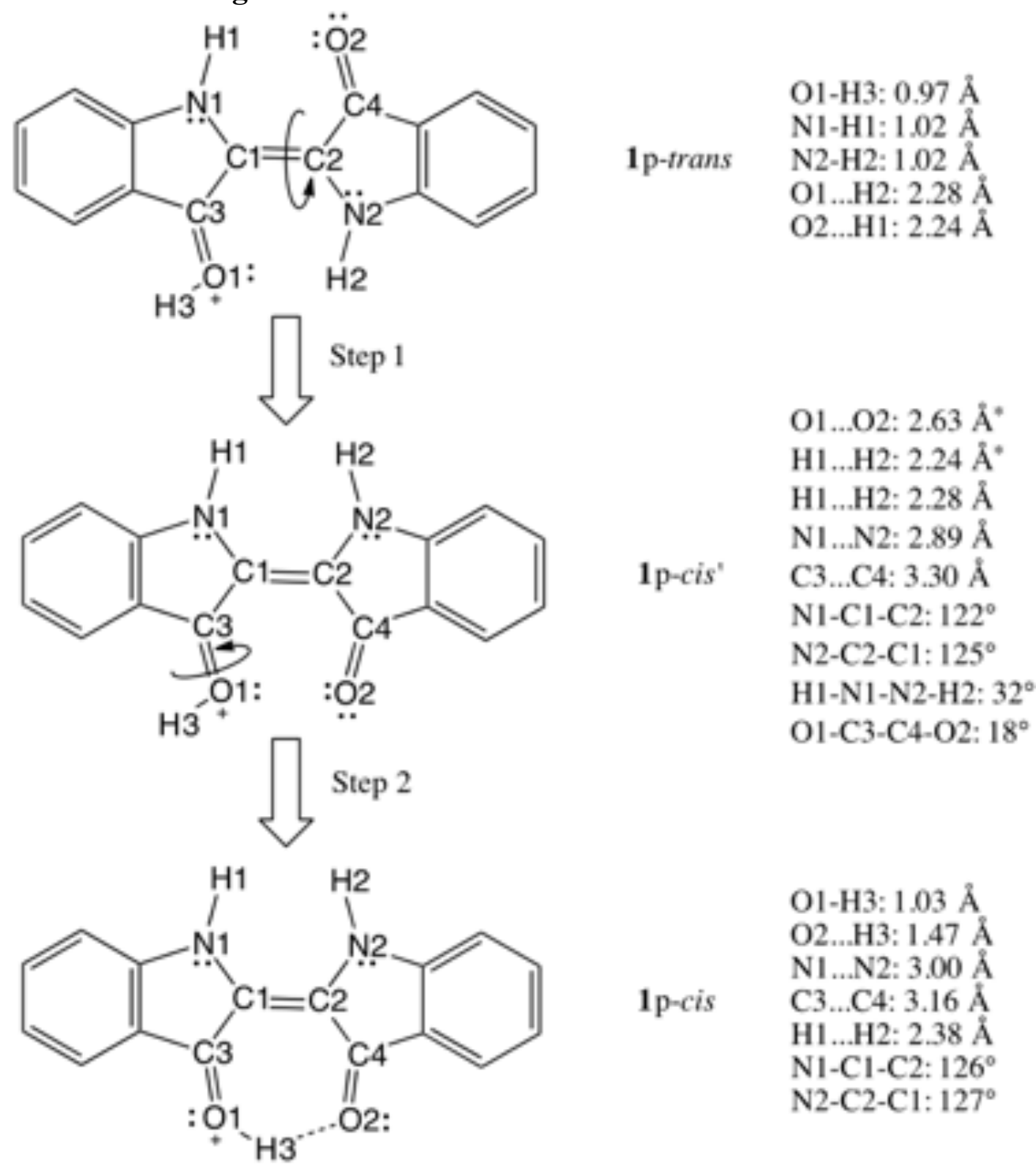

Scheme 2 Isomerization of protonated indigo. Important structural parameters that are discussed in the text are listed on the right of each molecule. The two asterisked parameters are for the unprotonated counterpart.

The optimized trans protonated and cis protonated indigo (1p-trans and 1p-cis, Scheme 2) structures are of $C_{s}$ symmetry. Attaching the proton $\mathrm{H} 3$ to the $\mathrm{O}$ atom is lower in energy by $0.39 \mathrm{eV}$ than attaching to the indole $\mathrm{N}$ atom. This is reasonable as forming the in-plane $\mathrm{OH}$ bond does not impair the extended conjugated $\pi$ system, while forming the out-of-plane NH bond does. In 1p-trans, the newly formed $\mathrm{OH}$ bond points away 
from the 3-oxindole unit on the other side of the central $\mathrm{C}=\mathrm{C}$ bridge, so that the steric hindrance between $\mathrm{H} 2$ and $\mathrm{H} 3$ is avoided. In 1p-cis, the $\mathrm{OH}$ bond points towards the opposite 3-oxindole to form an intramolecular hydrogen bond. This $\mathrm{O} 1-\mathrm{H} 3 \ldots \mathrm{O} 2$ hydrogen bond features a $1.03 \AA \mathrm{O}-\mathrm{H}$ distance and a $1.47 \AA \mathrm{H}$...O distance. It is this hydrogen bond that gives the planar structure for $1 \mathrm{p}$-cis. For the cis configuration with the $\mathrm{O} 1-\mathrm{H} 3$ bond pointing away from O2 (1p-cis', the intermediate cis structure, Scheme 2), a nonplanar structure is obtained, due to the steric hindrance between the two indoletype $\mathrm{H}$ atoms $(\mathrm{H} 1$ and $\mathrm{H} 2)$ and the Pauli repulsion between the lone pairs on the two $\mathrm{O}$ atoms. The H1-N1-N2-H2 and O1-C3-C4-O2 dihedral angles are 32 and 18 , respectively. If the structure is constrained to be planar, the $\mathrm{H} 1-\mathrm{H} 2$ and $\mathrm{O} 1-\mathrm{O} 2$ distances are 2.24 and $2.63 \AA$, shorter than twice the $\mathrm{H}$ and $\mathrm{O}$ van der Waals radii (1.20 and 1.52 $\AA),{ }^{33}$ respectively. The steric hindrance and the Pauli repulsion ensue. The presence of the $\mathrm{H} 3$ proton converts the $\mathrm{O}-\mathrm{O}$ Pauli repulsion to a hydrogen bond attraction. This hydrogen bond also bends the molecular framework so that the N1-C1-C2 and N2-C2-C1 angles are slightly increased from $122^{\circ}$ and $125^{\circ}$ in $1 \mathrm{p}$-cis' to $126^{\circ}$ and $127^{\circ}$ in $1 \mathrm{p}$-cis, respectively. As manifestations of the bending, the N1-N2 distance is increased from 2.89 $\AA$ in $1 \mathrm{p}$-cis' to $3.00 \AA$ in $1 \mathrm{p}$-cis, and the C3-C4 distance is reduced from 3.30 to $3.16 \AA$. The bending enlarges the $\mathrm{H} 1-\mathrm{H} 2$ distance to $2.38 \AA$, close to twice the $1.20 \AA$ van der Waals radius of $\mathrm{H}$, keeping them in the molecular plane. Their steric hindrance is hence eliminated, bringing about the coplanar structure of 1p-cis. In 1p-cis', the $2.28 \AA \mathrm{H} 1-\mathrm{H} 2$ distance is only achieved when they are on two sides of the molecular plane, i.e. with the pyramidalization of $\mathrm{N} 1$ and N2.

The $\mathrm{O} 1-\mathrm{H} 3 \ldots \mathrm{O} 2$ is a very strong hydrogen bond, reflected by the elongation of the $\mathrm{O} 1-\mathrm{H} 3$ bond from the typical $0.97 \AA$ in $1 \mathrm{p}$-trans to $1.03 \AA$ in $1 \mathrm{p}$-cis. This is a significant elongation. In comparison, the $\mathrm{OH}$ bond is not elongated in water dimer, which is bound by the significant $0.22 \mathrm{eV}$ hydrogen bond. ${ }^{34}$ The $1.47 \AA \mathrm{H} 3 . . \mathrm{O} 2$ distance is also substantially shorter than the $1.9 \AA^{35,36} \mathrm{H}$... O distance in liquid water. In comparison, the $\mathrm{N} 1-\mathrm{H} 1 \ldots \mathrm{O} 2$ and $\mathrm{N} 2-\mathrm{H} 2 \ldots \mathrm{O} 1$ hydrogen bonds in 1p-trans are much 
weaker. The $\mathrm{N}-\mathrm{H}$ bond length is $1.02 \AA$, not elongated from that of a single 3-oxindole unit by the N-H...O hydrogen bonding. Also, the H...O distance is as large as $2.28 \AA$.

In another limiting point of view, the $\mathrm{O} 1-\mathrm{H} 3 \ldots \mathrm{O} 2$ interaction of $1 \mathrm{p}$-cis can be understood as a 3-center-4-electron $(3 \mathrm{c} 4 \mathrm{e})$ covalent interaction, with the electrons coming from the two lone pairs of the $\mathrm{O}$ atoms and the proton forming the third center. This explains the severely elongated $\mathrm{O}-\mathrm{H}$ and short $\mathrm{H}$... O distances. Given the energy lowering associated with the hydrogen bond, $1 \mathrm{p}$-cis is more stable than $1 \mathrm{p}$-trans by 0.11 $\mathrm{eV}$. For comparison, 1-cis is higher in energy than 1-trans by $0.38 \mathrm{eV}$ according to our calculation. Evidently, the protonation is the thermodynamics trigger for the trans-to-cis conversion.

With this energy lowering, the isomerization of the protonated indigo is thermodynamically favorable. However, it takes two steps (Scheme 2) and the first step from $1 \mathrm{p}$-trans to $1 \mathrm{p}$-cis', the actual isomerization step around the central $\mathrm{C}=\mathrm{C}$ bond, is energetically uphill by $0.29 \mathrm{eV}$. This endoergicity is attributed to the O-O lone pairs' Pauli repulsion and the (indole)H-H(indole) steric hindrance mentioned above, as well as losing the $\mathrm{N}-\mathrm{H} . . . \mathrm{O}$ hydrogen bonds in trans. These energy-raising factors are also applicable to the trans-to-cis isomerization of the unprotonated indigo and account for the comparable endoergicity ( 0.38 vs $0.29 \mathrm{eV})$. Yet the stabilizing $\mathrm{O}-\mathrm{H} \ldots \mathrm{O}$ hydrogen bond is not in place. The TS structure for Step 1 has an $87^{\circ}$ dihedral angle between the two 3oxindole units, close to $90^{\circ}$, as it should be for an olefin isomerization. Breaking the two $\mathrm{N}-\mathrm{H} . . . \mathrm{O}$ hydrogen bonds and the $\pi$ bond leads to a fairly high $(1.29 \mathrm{eV})$ barrier. The $\pi$ bond is largely broken as the natural orbitals that correlate to the $\pi$-bonding and $\pi^{*}$ antibonding orbitals of 1p-trans have occupancies close to 1 (1.18 and 0.82, Figure 1). This occupation scheme indicates $82 \%$ diradical character. ${ }^{37}$ With the unfavorable thermodynamics and kinetics, Step 1 is unlikely to occur.

For comparison, the barrier of Step 1 is lower than the one for the 1-trans to 1-cis isomerization, which is calculated to be $1.54 \mathrm{eV}$. A possible reason for the lower barrier is that one N-H...O hydrogen bond (N2-H2 ..O 1 in Scheme 2) is weakened by the newly formed $\mathrm{O}-\mathrm{H}$ bond (O1-H3 in Scheme 2), so that the trans configuration is less stable in 
1p-trans than in 1-trans. When attached by the $\mathrm{H} 3$ proton, the $\mathrm{O} 1$ should have its lone pair lower in energy, and consequently diminish its ability as a hydrogen bond acceptor. The $\mathrm{O} 1 \ldots \mathrm{H} 2$ distance in 1p-trans is $2.28 \AA$, slightly longer than the $2.24 \AA \mathrm{O} 2 \ldots \mathrm{H} 1$ distance, reflecting the weaker hydrogen bond in the former. Another reason is that the isomerization breaks the central $\mathrm{C}=\mathrm{C} \pi$ bond more completely in 1. Indigo's natural orbitals analogous to those in Figure 1 have occupancies 1.01 and 0.99, an almost complete diradical. When one side is protonated, the two $\pi$ orbitals of $1 \mathrm{p}$ are not symmetrically distributed between $\mathrm{C} 1$ and $\mathrm{C} 2$ (vide infra). Some ionic character results and stabilizes the TS.

(a)

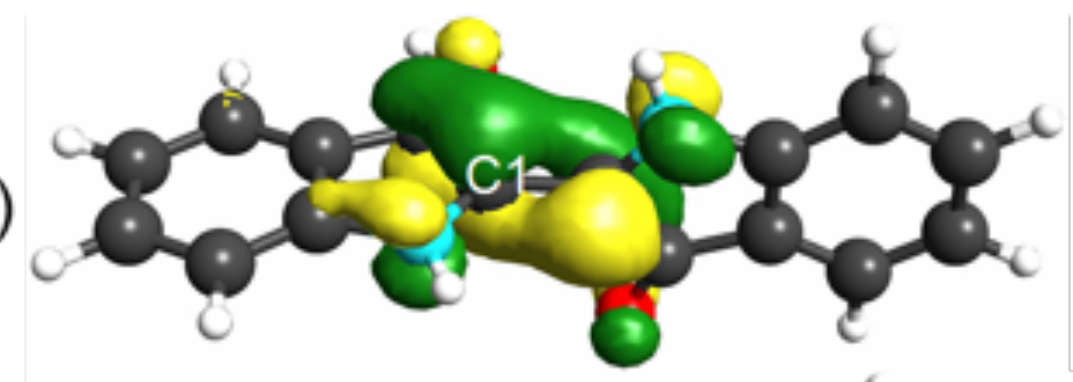

(b)

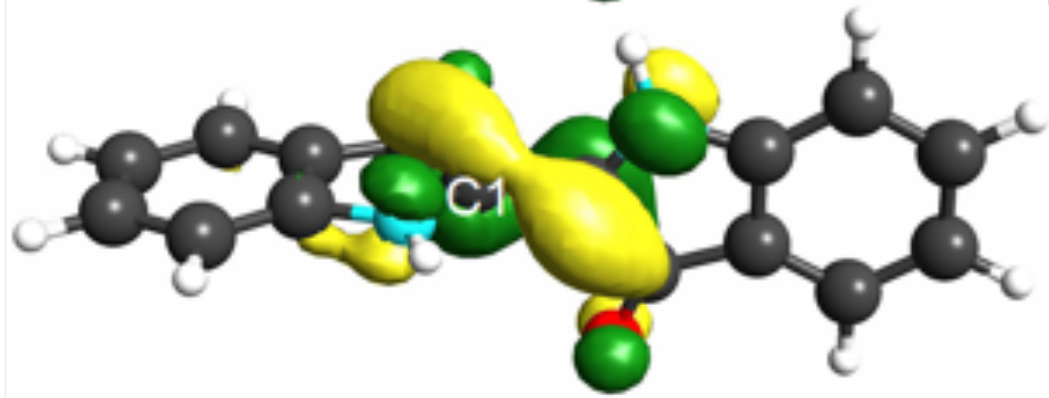

Figure 1 Natural orbitals of the TS in Step 1 of the isomerization of protonated indigo: (a) correlating to the central $\mathrm{C}=\mathrm{C} \pi$-bonding orbital in 1p-trans, occupancy $=1.18$; (b) correlating to the $\pi^{*}$-antibonding orbital, occupancy $=0.82 . \mathrm{H}, \mathrm{C}, \mathrm{N}$, and $\mathrm{O}$ atoms are represented by white, black, cyan, and red spheres, respectively. Only $\mathrm{C} 1$ is labelled as the other atom labels are clear based on the atoms' relative positions with respect to $\mathrm{C} 1$.

In Step 2, the proton $\mathrm{H} 3$ needs to migrate around the $\mathrm{C} 3=\mathrm{O} 1$ bond to attain $1 \mathrm{p}$-cis. The energy lowering in this proton migration is $0.40 \mathrm{eV}$, which makes the overall isomerization slightly exoergic (by $0.11 \mathrm{eV}$ ). The proton migration about the $\mathrm{C}=\mathrm{O}$ bond 
needs to overcome $\mathrm{a} \sim 0.30 \mathrm{eV}$ barrier. Note that this barrier is estimated by potential energy curve scanning along the $\mathrm{O} 1-\mathrm{H} 3$ bond rotation about the $\mathrm{C} 3=\mathrm{O} 1$ bond; no TS optimization was carried out. The actual barrier should be lower. The relatively low barrier is due to the small rearrangement of the electronic structure in this migration. As the $\mathrm{O}-\mathrm{H}$ bond rotates from pointing away from the opposite unit, to being perpendicular to the molecular plane, and then to pointing towards the opposite unit, the original $\mathrm{OH}$ bond pair becomes a lone pair, the $\mathrm{CO} \pi$ bond pair converts to the $\mathrm{OH}$ bond pair at the TS configuration, and then changes back to a $\mathrm{CO}$ bond pair later, and the $\mathrm{O}$ lone pair pointing towards the opposite unit becomes an $\mathrm{OH}$ bond pair (Scheme 3). The three pairs maintain being "pairs" during the migration. The overall electronic structure rearrangement is mild, not like converting the $\pi$ bond pair to a diradical in Step 1.
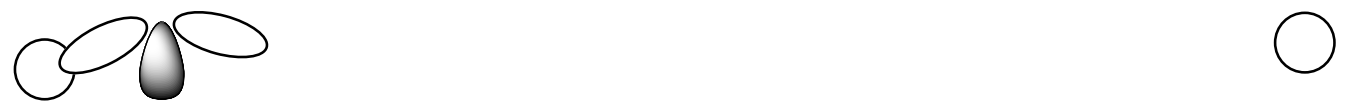

Scheme 3 Rearrangement of electronic structure in Step 2 of indigo protoisomerization

Given its exoergicity and low barrier, and the light mass of the proton, Step 2 should occur easily, if it were an isolated process. However, it is subsequent to Step 1. The stepwise nature of the isomerization is strengthened by the large difference in frequency between the respectively relevant vibrational modes. In 1p-trans, the normal modes corresponding to the central $\mathrm{C}=\mathrm{C}$ bond torsional motion and the $\mathrm{OH}$ bond rotation about the $\mathrm{C}=\mathrm{O}$ bond have wave numbers 70 and $641 \mathrm{~cm}^{-1}$, respectively; the coupling between the two motions should not be substantial. This is reasonable considering the large difference in masses of the two modes. According to Carpenter's Dynamic Matching picture, ${ }^{38}$ once the torsional motion has high enough kinetic energy in the $\mathrm{C}=\mathrm{C}$ torsional motion to overcome the $1.29 \mathrm{eV}$ barrier and reach the $1 \mathrm{p}$-cis' intermediate, it is 
likely to continue rotating in the same direction, bringing the intermediate forward to the original 1p-trans configuration. "Forward to" is used instead of "back to", as there is no need to bounce back, a feature of rotational isomerization. Such a one-way trans-cistrans conversion is schematically shown in Figure 2 (a). Since the 1p-cis' intermediate is less stable than 1p-trans, thermalization facilitates the forward-to-trans conversion, reducing the probability of having Step 2 occur. The reason for why indigo does not undergo protoisomerization under acidic conditions is now clear.
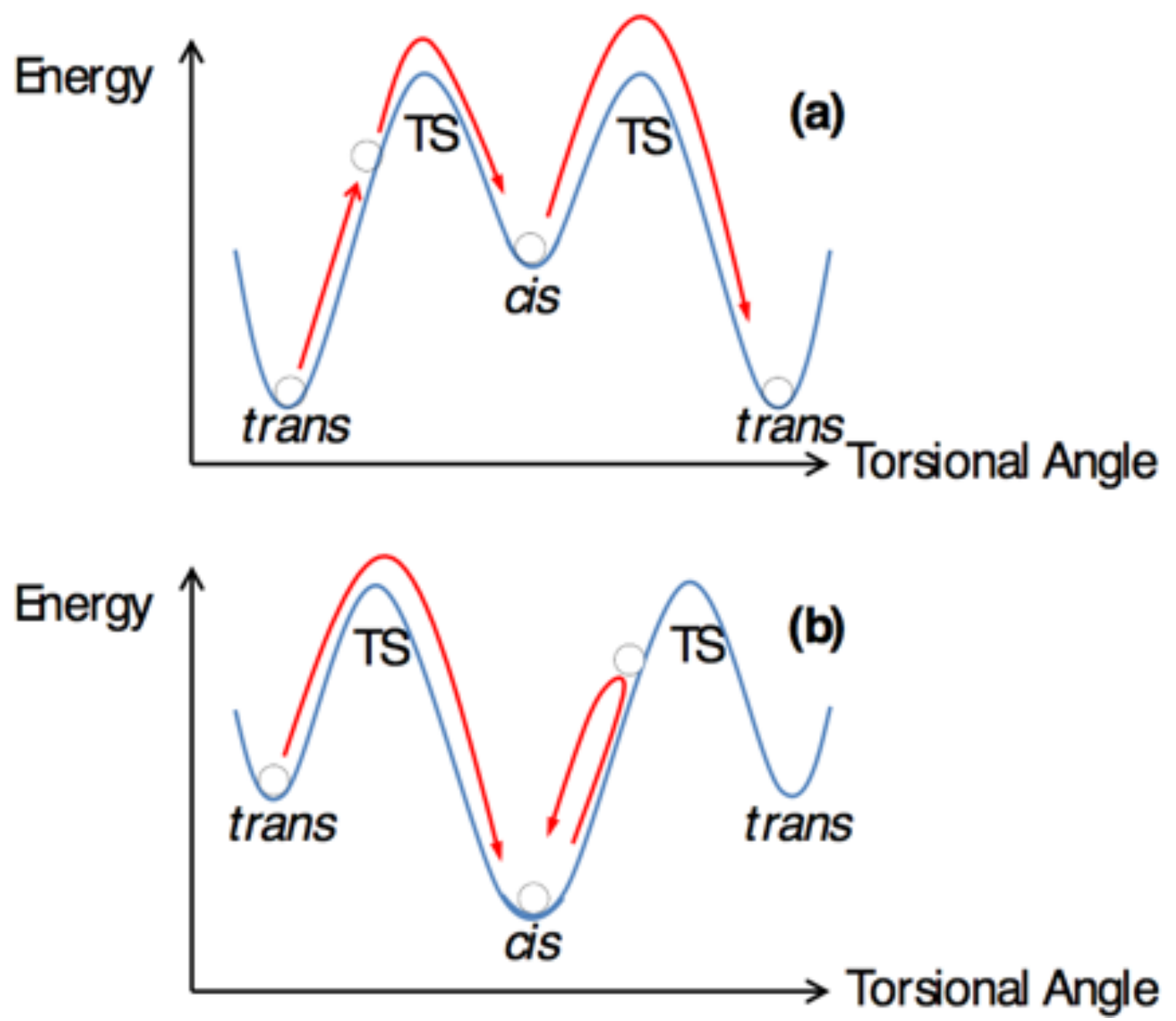

Figure 2 Sketches of the torsional motion around the central $\mathrm{C}=\mathrm{C}$ bond in the (a) endoergic and (b) exoergic trans-cis isomerization. Note that for the protonated indigo, the cis here refers to the $\mathbf{1} \mathrm{p}$-cis' intermediate, rather than the more stable $1 \mathrm{p}$-cis final 
product. The torsional motion has been analogized to the motion of a sphere along the respective characteristic potential energy curves. 


\section{Protonated Nindigo}

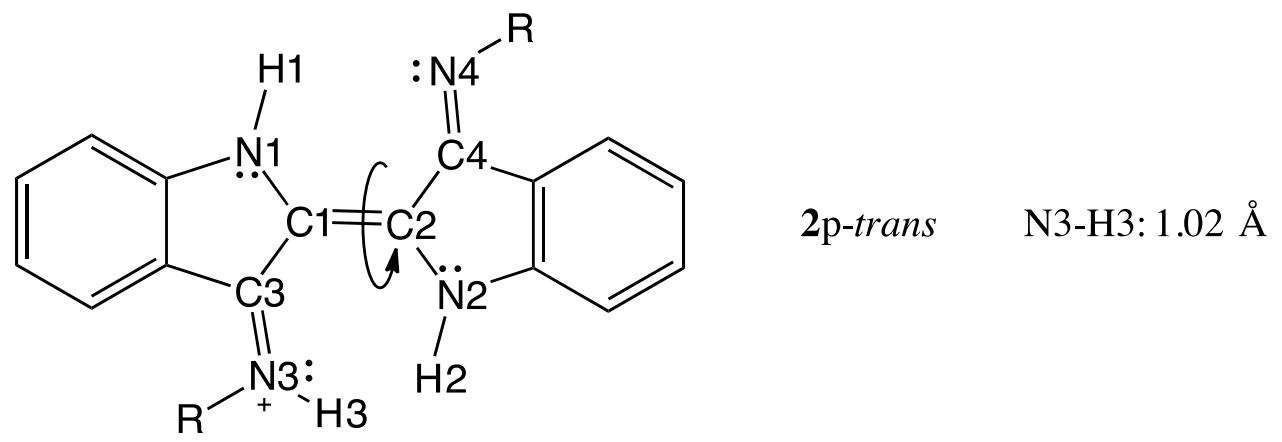

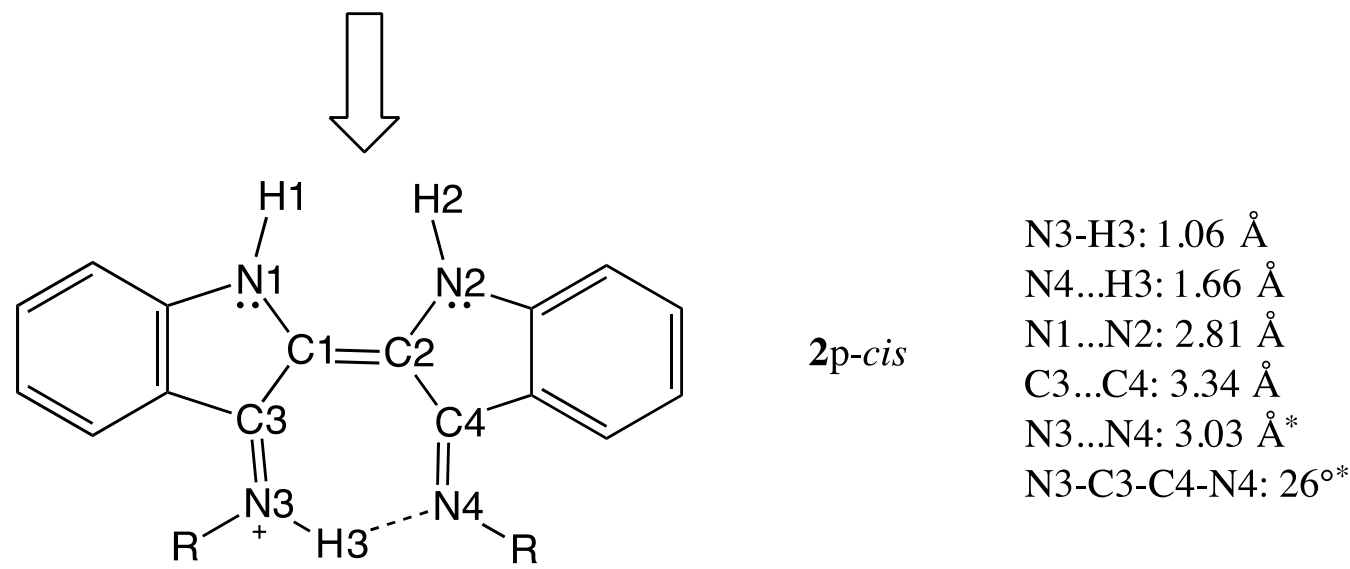

Scheme 4 Isomerization of protonated Nindigo. Important structural parameters that are discussed in the text are listed on the right of each molecule. The two asterisked parameters are for the unprotonated counterpart.

The optimized structures of protonated Nindigo, 2p-trans and 2p-cis (Scheme 4), are both of $C_{1}$ symmetry. The molecular frameworks are nonplanar even ignoring the $\mathrm{R}$ $\left(\mathrm{CH}_{3}{ }^{-}\right)$groups attached to the imine $\mathrm{N}$ atoms. In $2 \mathrm{p}$-trans, the protonic $\mathrm{H} 3$ is close to the indole-type $\mathrm{H} 2$. The resultant steric hindrance twists the structure to be nonplanar. In $2 \mathrm{p}$ cis, the steric hindrance between $\mathrm{H} 1$ and $\mathrm{H} 2$ forces them to be out of plane. Note that the $\mathbf{2 p}$-cis units in the solid extracted from the solution of trifluoroacetic acid and $\mathbf{2}$ exhibit a planar framework. ${ }^{20}$ This is because the two indole $\mathrm{H}$ atoms are drawn back to the plane by the trifluoroacetate counteranion that form hydrogen bonds with them. A similar 
planar structure is obtained in our optimization for $2 \mathrm{p}$-cis with a $\mathrm{Cl}^{-}$counteranion. The structure is shown in Figure S5 in SI.

The N-H...N bond features a $1.66 \AA \mathrm{H} . . \mathrm{N}$ distance, way shorter than the counterpart (2.36 $\AA$ ) in phase I solid ammonia. ${ }^{39}$ The N-H bond is elongated from $1.02 \AA$ in 2 -trans to $1.06 \AA$ in 2 p-cis. The smaller elongation (0.04 vs $0.06 \AA)$ suggests that the $\mathrm{N}-\mathrm{H} . . \mathrm{N}$ hydrogen bond is weaker than the $\mathrm{O}-\mathrm{H} \ldots \mathrm{O}$ in $1 \mathrm{p}$-cis. As a result of the weaker hydrogen bond and the intrinsically longer N3-H3 bond, the molecular framework of $2 \mathrm{p}-$ cis is not bent like 1p-cis: the N1-N2 and C3-C4 distances are 2.81 and $3.34 \AA$, similar to those in 1 p-cis'. Therefore, H1 and H2 in 2 p-cis need to adopt an out-of-plane configuration to avoid their steric hindrance.

$2 \mathrm{p}$-cis is lower in energy than $2 \mathrm{p}$-trans by $0.23 \mathrm{eV}$. The TS between them features a $76^{\circ}$ dihedral angle between the two NR-substituted-oxindole units and a $1.07 \mathrm{eV}$ barrier. The lower barrier (1.07 vs $1.29 \mathrm{eV})$ and larger exoergicity $(0.23 \mathrm{vs} 0.11 \mathrm{eV})$ immediately indicate that Nindigo undergoes protoisomerization more easily than indigo. The isomerization process of the protonated Nindigo is schematically shown in Figure 2(b). Once the complex gains enough kinetic energy to overcome the barrier, the deeper potential well on the cis side will trap the complex, with the kinetic energy dissipated by thermalization.

Further, the isomerization product $\mathbf{2 p}$-cis is obtained in one step, vs two steps for indigo. This difference is rooted in the different structures of Nindigo and indigo. In indigo, the incoming proton can freely adopt an $\mathrm{OH}$ bond orientation that points away from the nearby indole-type $\mathrm{H}$ to avoid the $\mathrm{H}-\mathrm{H}(\mathrm{H} 2-\mathrm{H} 3$ in Scheme 2) steric hindrance. But such an orientation has been taken by the NR in Nindigo. Therefore, the proton H3 can only form an $\mathrm{NH}$ bond pointing towards the opposite side of the central $\mathrm{C}=\mathrm{C}$ bond, which is ready to form the N-H...N hydrogen bond after isomerization. The so-induced $\mathrm{H} 2-\mathrm{H} 3$ hindrance forces $2 \mathrm{p}$-trans to adopt a nonplanar framework and raises its energy, contributing to the lower barrier and larger exoergicity. Using the Arrhenius equation and assuming equal pre-exponential factors, the $0.22 \mathrm{eV}$ lower barrier indicates that the 
isomerization of protonated Nindigo at room temperature $(298.15 \mathrm{~K})$ is $5.0 \times 10^{3}$ times faster than Step 1 in the isomerization of the protonated indigo.

The lower barrier is also related to the electronic structure of the TS between $2 \mathrm{p}$ trans and $\mathbf{2 p}$-cis. The TS's natural orbitals correlating to $2 \mathrm{p}$-trans's central $\mathrm{C}=\mathrm{C} \pi$ bonding and $\pi^{*}$-antibonding orbitals are shown in Figure 3. The occupancies deviate more from 1 than the counterparts of $1 \mathrm{p}$ (Figure 2). The bonding orbital is occupied by $\sim 1.5$ electrons while the antibonding orbital by $\sim 0.5$, i.e., less diradical character in the TS. A close inspection found that both orbitals have larger amplitudes on $\mathrm{C} 1$ (on the protonated side) than on $\mathrm{C} 2$. The Mulliken populations of the two orbitals on $\mathrm{C} 1$ are 0.35 and 0.10 , while those on $\mathrm{C} 2$ are 0.09 and 0.06 . In comparison, the distribution of the corresponding orbitals at the protonated indigo's TS (Figure 1) is more symmetrical, with the Mulliken populations of 0.24 and 0.14 on $\mathrm{C} 1$, and 0.33 and 0.26 on $\mathrm{C} 2$. The orbitals in the $2 \mathrm{p}$ TS are polarized towards the protonated side of the molecule. The occupation scheme and the polarization of the orbitals suggest that at the $2 p$ TS structure, the central $\mathrm{CC} \pi$ bond is not broken into two unpaired electrons (i.e., a diradical) as much as in the case for $1 \mathrm{p}$. Instead, it contains some ionic character to stabilize the structure, and hence lower the barrier. Considering that the TS structure is obtained by assuming a diradical TS state, i.e., with the triplet geometry optimization, it does not reflect the partially ionic electronic structure, and the so-obtained $1.07 \mathrm{eV}$ barrier should be taken as an overestimation. 


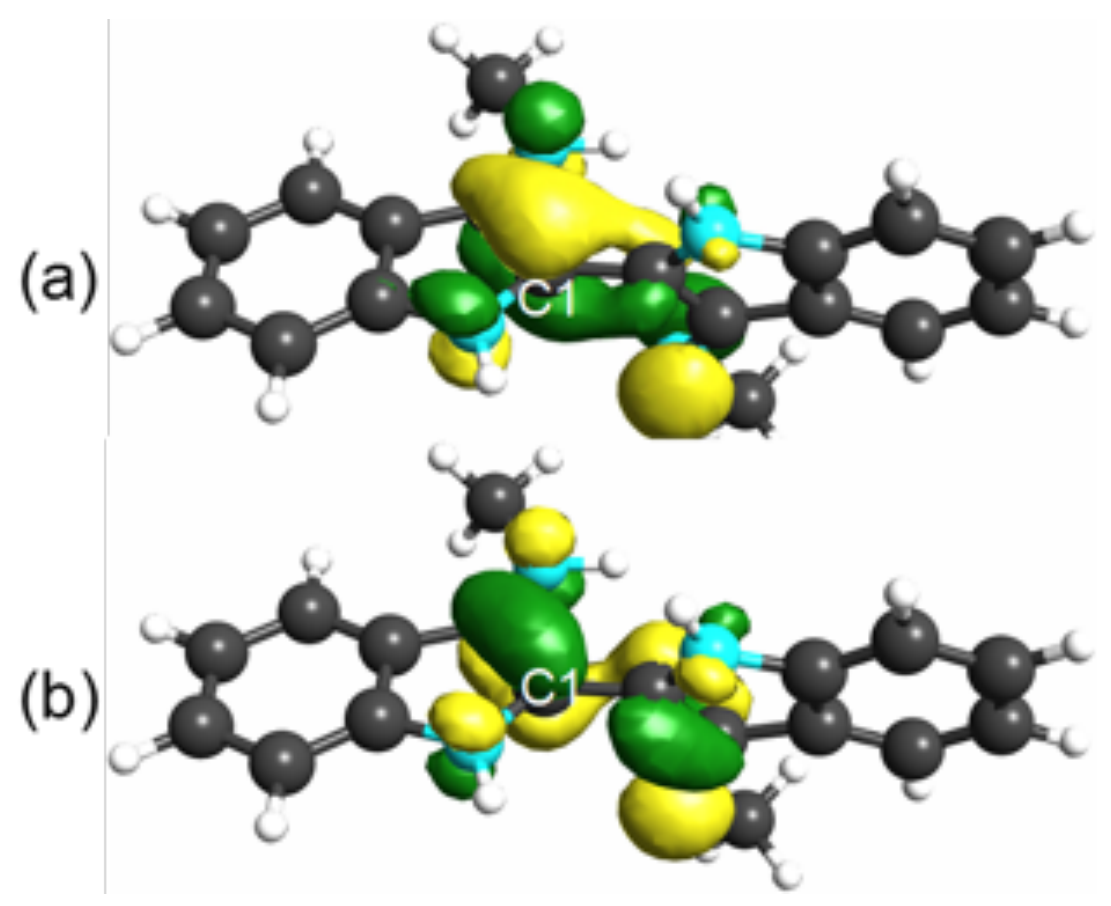

Figure 3 Natural orbitals of Nindigo's TS: (a) correlating to the central $\mathrm{C}=\mathrm{C} \pi$-bonding orbital of $2 \mathrm{p}$-trans, occupancy $=1.47$; (b) correlating to the $\pi^{*}$-antibonding orbital, occupancy $=0.53$. H, C, and $\mathrm{N}$ atoms are represented by white, black, and cyan spheres. Only $\mathrm{C} 1$ is labelled as the other atom labels are clear based on the atoms' relative positions with respect to $\mathrm{C} 1$.

Overall, the larger exoergicity, the lower barrier, and the one-step process lead to the facile protoisomerization of Nindigo. The specific orientation of the NH bond formed in protonating Nindigo contributes to all these factors.

The proton's role in the isomerization is even more obvious when we compare $2 p$ and 2. The barrier for 2-trans to 2-cis isomerization is increased to $1.41 \mathrm{eV}$, given the more significant diradical character at its TS (occupancies 1.11 and 0.89 in the two orbitals analogous to those in Figure 3). The more striking difference is in the isomerization energy: 2-cis is higher in energy than 2-trans by $0.87 \mathrm{eV}$. Again, the endoergicity is largely attributed to the Pauli repulsion between the lone pairs of the two imine $\mathrm{N}$ atoms. To reduce the Pauli repulsion, the molecular framework (shown in Figure 
$\mathrm{S} 3$ in SI) has to adopt a nonplanar structure with the $26^{\circ} \mathrm{N} 3-\mathrm{C} 3-\mathrm{C} 4-\mathrm{N} 4$ dihedral angle so that the N3-N4 distance is increased to $3.03 \AA$, close to twice the van der Waals radius of $\mathrm{N}(1.55 \AA) .{ }^{42}$ The role of proton in the isomerization, especially in converting the Pauli exclusion between the two imine $\mathrm{N}$ atoms (or carbonyl $\mathrm{O}$ atoms) to a strong hydrogen bond attraction, cannot be over-emphasized.

\section{Protonated Indigo Monoimine}

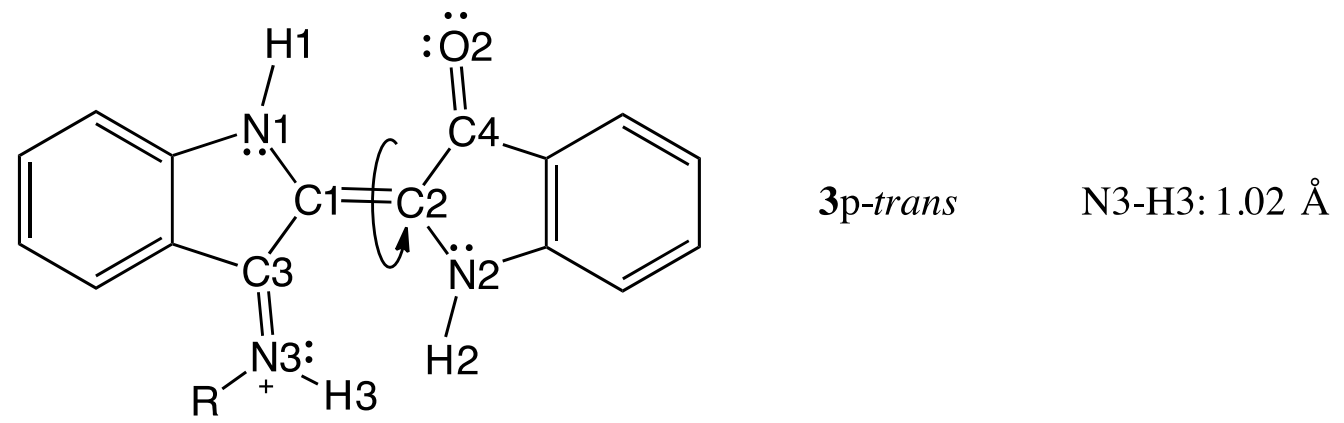

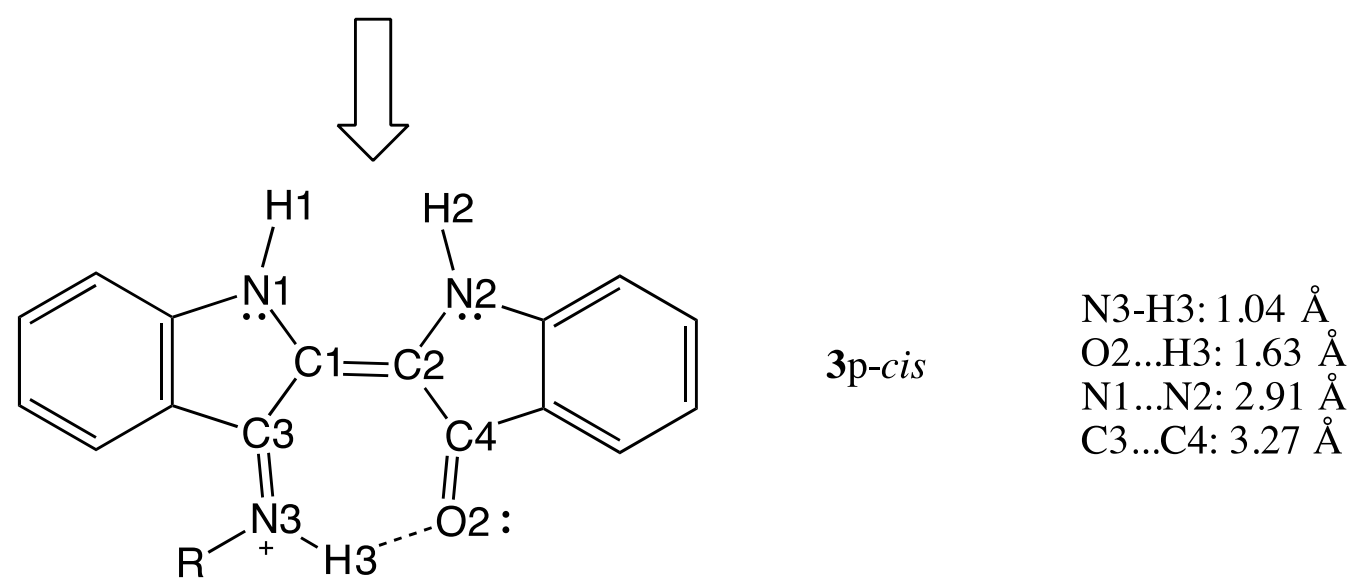

Scheme 5 Isomerization of protonated indigo monoimine. Important structural parameters that are discussed in the text are listed on the right of each molecule.

In the last case, we investigate the protoisomerization of indigo monoimine (Scheme 5), in which only one 3-oxindole unit of indigo has its carbonyl group replaced by an imine. ${ }^{20}$ This molecule is the "middle ground" between indigo and Nindigo and will offer insight into the differences between them. Our calculations show that the protonation on the imine $\mathrm{N}$ is more favorable than on the carbonyl $\mathrm{O}$ by $0.18 \mathrm{eV}$, consistent with the fact 
that the three-coordinated $\mathrm{N}$ with a lone pair (ammonia, imine, etc.) is more basic than the two-coordinated $\mathrm{O}$ with two lone pairs (water, carbonyl, etc.). The preference towards the imine site is strong enough to overcome the $\mathrm{H} 2-\mathrm{H} 3$ steric hindrance, which would not be present if $\mathrm{H} 3$ attacked the oxygen. The hindrance leads to a non-planar structure for 3p-trans.

In 3p-cis, the $\mathrm{N} 3-\mathrm{H} 3 \ldots \mathrm{O} 2$ interaction features a $1.04 \AA \mathrm{N}-\mathrm{H}$ distance and a 1.63 $\AA$ H...O distance. Compared to the counterpart in 3p-trans, the N3-H3 bond is only elongated by $0.02 \AA$. This is smaller than the $0.06 \AA$ elongation of the $\mathrm{O}-\mathrm{H}$ from $1 \mathrm{p}$-trans to $\mathbf{1 p}$-cis. Also, the H...O distance in $\mathbf{3 p}$-cis is significantly longer than the $1.47 \AA$ counterpart in 1p-cis. The $\mathrm{N}-\mathrm{H} . . . \mathrm{O}$ interaction is hence weaker than the $\mathrm{O}-\mathrm{H} . . . \mathrm{O}$ interaction in $1 \mathrm{p}$-cis. As the imine $\mathrm{N}$ is a stronger base than the carbonyl $\mathrm{O}$, the incoming proton forms a stronger $\mathrm{NH}$ bond in $3 \mathrm{p}$ than the $\mathrm{OH}$ bond in $1 \mathrm{p}$. The former is then a worse hydrogen bond donor and forms a weaker hydrogen bond, given the same accepter carbonyl $\mathrm{O}$ in both cis configurations. The weaker hydrogen bond does not bend the molecular framework as in 1p-cis. The N1-N2 and C3-C4 distances are 2.91 and $3.27 \AA$, similar to those of $1 \mathrm{p}$-cis'. Likewise, $\mathrm{H} 1$ and $\mathrm{H} 2$ repel each other to be out of plane of the molecular framework.

The $3 \mathrm{p}$-trans to $3 \mathrm{p}$-cis isomerization is exoergic by $0.33 \mathrm{eV}$ and needs to overcome a $1.15 \mathrm{eV}$ barrier. It is both thermodynamically and kinetically more favorable than the isomerization of the unprotonated $\mathbf{3}$, which features $0.67 \mathrm{eV}$ endoergicity and a $1.48 \mathrm{eV}$ barrier. The TS has an $82^{\circ}$ dihedral angle between the oxindole and substituted oxindole units. Similar to $2 \mathrm{p}$, the orientation of the N3-H3 bond in $3 p$ leads to a one-step isomerization.

The TS's natural orbitals correlating to the central $\mathrm{C}=\mathrm{C} \pi$-bonding and $\pi^{*}$ antibonding orbitals are shown in Figure 4. The occupancies are close to those in 1p, with a pronounced diradical character. The ionicity of the $\pi$ interaction is not obvious, as the Mulliken populations of the bonding orbital on $\mathrm{C} 1$ and $\mathrm{C} 2$ are 0.26 and 0.34 , respectively, while those of the antibonding orbital are 0.17 and 0.25 . These populations are very close to the protonated indigo counterparts, in combination with the similar orbital 
occupancies, suggesting a similar electronic structure. With the need to break the $\mathrm{CC} \pi$ bond to a larger extent, the barrier is higher than that of protonated Nindigo (1.15 vs 1.07 $\mathrm{eV})$. On the other hand, with the $\mathrm{H} 2-\mathrm{H} 3$ hindrance raising $\mathbf{3 p}$-trans's energy, the barrier is lower than the $1.29 \mathrm{eV}$ of $1 \mathrm{p}$. The moderate barrier, and the one-step isomerization explain the observed protoisomerization of indigo monoimine. ${ }^{20}$ All calculated isomerization energies and barriers for the three protonated cases and their unprotonated counterparts are summarized in Table 2 for comparison.

(a)

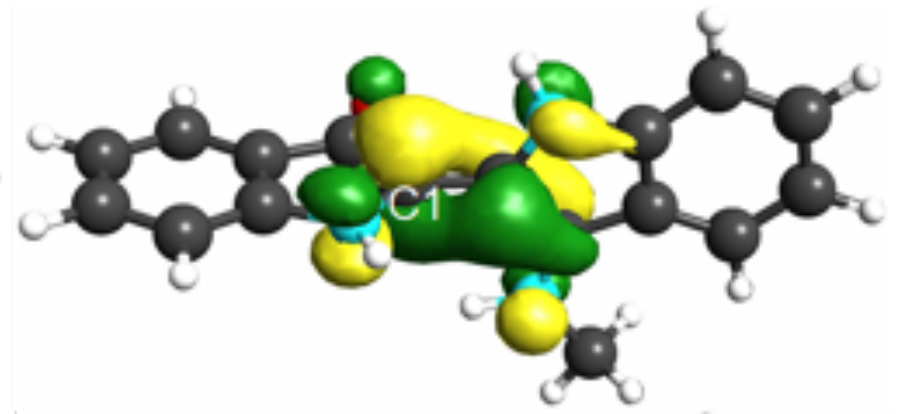

(b)

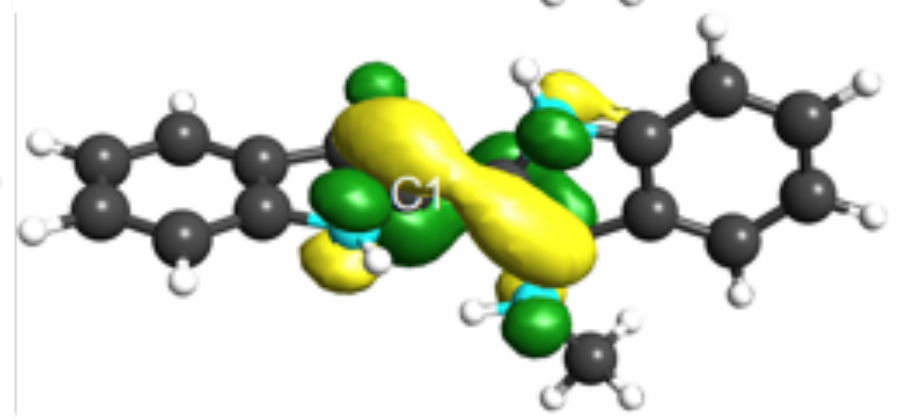

Figure 4 Natural orbitals of monoimine TS: (a) correlating to the central $\mathrm{C}=\mathrm{C} \pi$-bonding orbital of $3 p$-trans, occupancy $=1.18$; (b) correlating to the $\pi^{*}$-antibonding orbital, occupancy $=0.82 . \mathrm{H}, \mathrm{C}, \mathrm{N}$, and $\mathrm{O}$ atoms are represented by white, black, cyan, and red spheres. Only $\mathrm{C} 1$ is labeled as the other atom labels are clear based on the atoms' relative positions with respect to $\mathrm{C} 1$.

Table 2 Isomerization Energies and Barriers (both in eV) of the Investigated Molecules. Negative isomerization energy means the cis configuration is more stable. Molecule Isomerization Energy Barrier 


\begin{tabular}{ccc}
\hline $\mathbf{1} \mathrm{p}^{\mathrm{a}}$ & -0.11 & 1.29 \\
$\mathbf{2 p}$ & -0.23 & 1.07 \\
$\mathbf{3 p}$ & -0.33 & 1.15 \\
$\mathbf{1}$ & 0.38 & 1.54 \\
$\mathbf{2}$ & 0.87 & 1.41 \\
$\mathbf{3}$ & 0.67 & 1.48 \\
\hline
\end{tabular}

${ }^{a}$ For the protonated indigo, the isomerization energy is the energy difference between $\mathbf{1 p}$ cis and $1 \mathrm{p}$-trans. The barrier is for Step 1, from $1 \mathrm{p}$-trans to $1 \mathrm{p}$-cis'.

\section{CONCLUSION}

Through the electronic structure calculations, the reason behind the different trans-to-cis protoisomerization propensities of indigo and its mono and diimine derivatives is clarified. The protoisomerizations of all three molecules are exoergic. The kinetics makes the difference, and it is mostly because of the different orientations of the $\mathrm{OH}$ and $\mathrm{NH}$ bond formed in protonation. The $\mathrm{OH}$ bond formed in protonating indigo points away from the other oxindole unit to avoid H-H steric hindrance. Such an orientation requires a two-step isomerization with a high energy intermediate (1p-cis'), which is likely to carry on along the torsional motion forward to the trans structure. Also, the central CC $\pi$ bond is largely broken to a singlet diradical electronic structure at the transition state to attain the intermediate. The resultant high barrier further hinders the isomerization. Indigo hence does not undergo protoisomerization.

The protonated Nindigo, with its protonation-formed NH bond pointing towards the other substituted oxindole unit, features a one-step isomerization. Furthermore, during the rotation about the central $\mathrm{CC}$ bond, the $\mathrm{CC} \pi$ bond is partially polarized to retain some ionic interaction, instead of being broken into a diradical. The associated lowering of the energy barrier facilitates the isomerization. The protonated monoimine is the in-between case of indigo and Nindigo. Its trans 
configuration is similar to the protonated Nindigo, and hence a one-step isomerization ensues. On the other hand, the transition state in its isomerization displays a significant diradical character, similar to the protonated indigo. The energy barrier is hence higher than that of the protonated Nindigo. The fact that the protonated monoimine still undergoes the isomerization indicates that the number of steps is the determining factor.

\section{ASSOCIATED CONTENT}

\section{Supporting Information}

Coordinates of all reported structures; comparison between $\mathrm{R}=$ methyl and phenyl for $\mathbf{2}$ and $2 \mathrm{p} ; 2 \mathrm{p}$-cis structure with a $\mathrm{Cl}^{-}$counteranion; fractionally occupied natural orbitals of $1 \mathrm{p}$ in its trans, cis, and TS configurations.

\section{AUTHOR INFORMATION}

Corresponding Author

*E-mail: toby.zeng@,carleton.ca

*Tel: 1-613-5202600 ext: 1647

Notes

The authors declare no competing financial interest.

\section{ACKNOWLEDGEMENTS}

Computations were made on the supercomputer Mammouth parallèle 2 from Université de Sherbrooke, managed by Calcul Québec and Compute Canada. We thank Mike Schmidt and Mark Gordon (Iowa State University) for their continuous support of the GAMESS-US program package. T.Z. thanks Natural Sciences of Engineering Research Council (NSERC) of Canada (RGPIN-2016-06276) and Carleton University (186853) for funding. R.G.H. thanks NSERC (RGPIN 5388-2014) and the University of Victoria for support. 


\section{REFERENCES}

${ }^{1}$ Zechmeister, L. Cis-Trans Isomerization and Stereochemistry of Carotenoids and Diphenyl-Polyenes. Chem. Rev. 1944, 34, 267-344.

${ }^{2}$ Cooper E. A.; Edgar, S. H. The Biological Significance of Cis-Trans Isomerism. Biochem. J. 1926, 20, 1060-1070.

${ }^{3}$ Brode W. R; Gould, J. H; Wyman, G. M. The Relation between the Absorption Spectra and the Chemical Constitution of Dyes. XXV. Phototropism and Cis-Trans Isomerism in Aromatic Azo Compounds. J. Am. Chem. Soc. 1952, 74, 4641.

${ }^{4}$ Haddow, A.; Harris, R. J. C; Kon, G. A. R; Roe, E. M. F. The Growth-Inhibitory and Carcinogenic Properties of 4-Aminostilbene and Derivatives. Phil. Trans. Roy. Soc. 1948, A241, 167.

${ }_{5}^{5}$ Seefelder, M. Indigo in Culture, Science, and Technology; Ecomed: Landsberg, Germany, 1994.

${ }^{6}$ Jacquemin, D.; Preat, J.; Wathelet, V.; Perpete, E. A. Substitution and Chemical Environment Effects on the Absorption Spectrum of Indigo. J. Chem. Phys. 2006, 124, 074104.

${ }^{7}$ Kobayashi, T.; Rentzepis, P. M. On the Picosecond Kinetics and Photostability of Indigo and 6,6'-Dimethoxyindigo. J. Chem. Phys. 1979, 70, 886-892.

${ }^{8}$ Miliani, C.; Romani, A.; Favaro, G. A Spectrophotometric and Fluorimetric Study of Some Anthraquinoid and Indigoid Colorants Used in Artistic Paintings. Spectrochim. Acta, Part A 1998, 54, 581-588.

${ }_{9}^{9}$ Moreno, M; Ortiz-Sanchez, J. M; Gelabert, R.; Lluch, J. M. A Theoretical Study of the Photochemistry of Indigo in its Neutral and Dianionic (Leucoindigo) Forms. Phys. Chem. Chem. Phys. 2013, 15, 20236-20246.

${ }^{10}$ Rondão, R.; de Melo, J. S.; Melo, M. J.; Parola, A. J. Excited-State Isomerization of Leuco Indigo. J. Phys. Chem. A 2012, 116, 2826-2832.

${ }^{11}$ Elsaesser, T.; Kaiser, W.; Lüttke, W. Picosecond Spectroscopy of Intramolecular Hydrogen Bonds in 4,4',7,7'-Tetramethylindigo. J. Phys. Chem. 1986, 90, 2901-2905.

${ }^{12}$ Cui G.; Thiel, W. Nonadiabatic Dynamics of a Truncated Indigo Model. Phys. Chem. Chem. Phys. 2012, 14, 12378-12384.

13 Yamazaki, S.; Sobolewski, A. L.; Domcke, W. Molecular Mechanisms of the Photostability of Indigo. Phys. Chem. Chem. Phys. 2011, 13, 1618-1628. 
${ }^{14}$ Nicholls-Allison E, C; Nawn, G; Patrick, B. O.; Hicks, R. G. Protoisomerization of Indigo di- and monoimines. Chem. Commun. 2015, 51, 12482-12485.

${ }^{15}$ Wyman, G. M.; Brode, W. R. The Relation Between the Absorption Spectra and the Chemical Constitution of Dyes. XXIV. Absorption Spectra of Some Thioindigo Dyes in Sulfuric Acid. J. Am. Chem. Soc. 1951, 73, 1487-1493.

${ }^{16}$ Maeda, Y.; Okada, T.; Mataga, N.; Irie, M. Picosecond Spectroscopy of transThioindigo and the Mechanism of Trans $\rightarrow$ Cis Photoisomerization. J. Phys. Chem. 1984, 88, 1117-1119.

${ }^{17}$ Gorner, H.; Pouliquen, J.; Kossanyi, J. Trans to Cis Photoisomerization of N,N'disubstituted Indigo Dyes via Excited Singlet States; A Laser Flash Photolysis and Steady State Irradiation Study. Can. J. Chem. 1987, 65, 708-717.

${ }^{18}$ Nawn, G; Waldie, K. M.; Oakley, S. R.; Peters, B. D.; Mandel, D.; Patrick, B. O.; Mcdonald, R.; Hicks, R. G. Redox-Active Bridging Ligands Based on Indigo Diimine ("Nindigo") Derivatives. Inorg. Chem. 2011, 50, 9826-9837.

${ }^{19}$ Fortier, S.; Moral, O. G.; Chen, C.-H.; Pink, M.; Le Roy, J. J.; Murugesu, M.; Mindiola, D. J.; Caulton, K. G. Probing the Redox Non-Innocence of Dinuclear, Three-Coordinate Co(II) Nindigo Complexes: Not Simply $\beta$-Diketiminate Variants. Chem. Commun. 2012, $48,11082-11084$.

${ }^{20}$ Mondal, P.; Ehret, F.; Bubrin, M.; Das, A.; Mobin, S. M.; Kaim, W.; Lahiri, G. K. A Diruthenium Complex of a "Nindigo" Ligand. Inorg. Chem. 2013, 52, 8467-8475.

${ }^{21}$ Mondal, P.; Plebst, S.; Ray, R.; Mobin, S. M.; Kaim, W.; Lahiri, G. K. Uncommon cis Configuration of a Metal-Metal Bridging Noninnocent Nindigo Ligand. Inorg. Chem. 2014, 53, 9348-9356.

${ }^{22}$ Shi, W.; Ma, H. Spectroscopic Probes with Changeable $\pi$-Conjugated Systems. Chem. Commun. 2012, 48, 8732-8744.

${ }^{23}$ Glowacki, E. D.; Voss, G.; Sariciftci, N. S. Progress in Chemistry and Applications of Functional Indigoes for Organic Electronics. Adv. Mater. 2013, 25, 6783-6799.

${ }^{24}$ Zhao, Y.; Truhlar, D. G. The M06 Suite of Density Functionals for Main Group Thermochemistry, Thermochemical Kinetics, Noncovalent Interactions, Excited States, and Transition Elements: Two New Functionals and Systematic Testing of Four M06class Functionals and 12 Other Functionals. Theor. Chem. Acc. 2008, 120, 215-241.

${ }^{25}$ Bargon, J. The Inter-Relationship Between Triplet Energies and Spin Chemistry. Photochem. Photobiol. Sci., 2006, 5, 970-978. 
${ }^{26}$ Nakano, H.; Uchiyama, R. Relativistic Quasidegenerate Perturbation Theory with FourComponent General Multiconfiguration Reference Functions. J. Chem. Phys. 2006, 124, 044101.

${ }^{27}$ Ebisuzaki, R.; Watanabe, Y.; Nakano, H. Efficient Implementation of Relativistic and Non-Relativistic Quasidegenerate Perturbation Theory with General Multiconfigurational Reference Functions. Chem. Phys. Lett. 2007, 442, 164-169.

${ }^{28}$ Barone, V.; Cossi, M. Quantum Calculation of Molecular Energies and Energy Gradients in Solution by a Conductor Solvent Model. J. Phys. Chem. A 1998, 102, 1995-2001.

${ }^{29}$ Cossi, M.; Rega, N.; Scalmani, G.; Barone, V. Energies, Structures, and Electronic Properties of Molecules in Solution with the C-PCM Solvation Model. J. Comput. Chem. 2003, 24, 669-681.

${ }^{30}$ Schmidt, M. W.; Baldridge, K. K.; Boatz, J. A.; Elbert, S. T.; Gordon, M. S.; Jensen, J. H.; Koseki, S.; Matsunaga, N.; Nguyen, K. A.; Su, S. et al. General Atomic and Molecular Electronic Structure System. J. Comput. Chem. 1993, 14, 1347-1363

${ }^{31}$ Gordon, M. S.; Schmidt, M. W. Advances in Electronic Structure Theory: GAMESS a Decade Later in Theory and Applications of Computational Chemistry: the First Forty Years; Dykstra, C. E.; Frenking, G. F.; Kim, K. S.; Scuseria, G. E., Eds.; Elsevier: Amsterdam, 2005; pp. 1167-1189.

${ }^{32}$ Dunning, T. H. Gaussian Basis Sets for Use in Correlated Molecular Calculations. I. The Atoms Boron Through Neon and Hydrogen. J. Chem. Phys. 1989, 90, 1007-1023.

${ }^{33}$ Bondi, A. van der Waals Volumes and Radii. J. Phys. Chem. 1964, 68, 441-451.

${ }^{34}$ Lane, J. R. CCSDTQ Optimized Geometry of Water Dimer. J. Chem. Theory. Comput. 2013, 9, 316-323.

${ }^{35}$ Modig, K.; Pfrommer, B. G.; Halle, B. Temperature-Dependent Hydrogen-Bond Geometry in Liquid Water. Phys. Rev. Lett. 2003, 90, 075502.

${ }^{36}$ Chaplin, M. Water's Hydrogen Bond Strength in Water and Life; Lynden-Bell, R. M.; Morris, S. C.; Barrow, J. D.; Finney, J. L.; Harper, C. L. Eds.; CRC Press: Boca Raton, 2010; pp. 69-86.

${ }^{37}$ Minami, T.; Ito, S.; Nakano, M. Theoretical Study of Singlet Fission in Oligorylenes. $J$. Phys. Chem. Lett. 2012, 3, 2719-2723.

${ }^{38}$ Carpenter, B. K. Dynamic Behavior of Organic Reactive Intermediates. Angew. Chem. Int. Ed. 1998, 37, 3340-3350. 
${ }^{39}$ Fortes, A. D.; Bordholt, J.P.; Wood, I. G.; Vocadlo, L. Hydrogen Bonding in Solid Ammonia from ab initio Calculations. J. Chem. Phys. 2003, 118, 5987-5994. 
Table of Contents Graphic

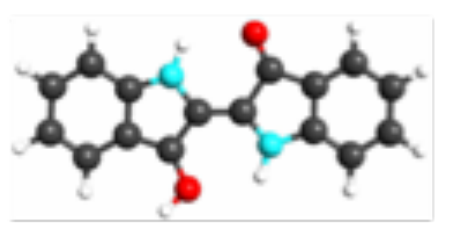

